\title{
Regard épistémologique sur la norme en anglais de spécialité : analyse exploratoire des registres au travers de deux ouvrages théoriques
}

An epistemological approach to the concept of norm in English for Specific

Purposes: An exploratory analysis of registers in two reference books

\section{Tiffany Jandrain}

\section{OpenEdition \\ Journals}

Édition électronique

URL : http://journals.openedition.org/asp/5080

DOI : $10.4000 /$ asp. 5080

ISSN : 2108-6354

\section{Éditeur}

Groupe d'étude et de recherche en anglais de spécialité

Édition imprimée

Date de publication : 1 novembre 2017

Pagination : 83-92

ISSN : 1246-8185

Référence électronique

Tiffany Jandrain, «Regard épistémologique sur la norme en anglais de spécialité : analyse exploratoire des registres au travers de deux ouvrages théoriques », ASp [En ligne], 72 | 2017, mis en ligne le 01 novembre 2018, consulté le 05 novembre 2020. URL : http://journals.openedition.org/asp/5080 ; DOI : https://doi.org/10.4000/asp.5080

Ce document a été généré automatiquement le 5 novembre 2020.

Tous droits réservés 


\section{Regard épistémologique sur la norme en anglais de spécialité : analyse exploratoire des registres au travers de deux ouvrages théoriques}

An epistemological approach to the concept of norm in English for Specific Purposes: An exploratory analysis of registers in two reference books

Tiffany Jandrain

\section{Introduction}

1 La langue varie selon de multiples facteurs. Parmi ceux-ci, la situation de communication détient indéniablement une place capitale. À l'origine de nombreuses analyses et discussions, cette variation entraînée par le contexte situationnel et définissant l'utilisation de la langue a été envisagée sous divers angles d'approche et diverses appellations. Fondée en 1968 par Michael A.K. Halliday, la théorie de la linguistique systémique fonctionnelle («SFL » pour Systemic Functional Linguistics), dont la ligne conductrice consiste à étudier la langue comme moyen de communication en véhiculant du sens en fonction du contexte (Munday 2016: 142), s'est longuement penchée sur cette variation contextuelle, dont elle a dénommé la variété de langue associée "registre », au point de devenir une approche théorique maîtresse dans son étude.

Dans un article sur les approches linguistiques en anglais de spécialité, Christopher Gledhill et Natalie Kübler (2016) avancent que l'anglais de spécialité (ASP), qu'ils conçoivent comme « une configuration spécifique de la langue anglaise utilisée par un groupe de spécialistes en vue de répondre à des objectifs de ce groupe » (2016:66, 
notre traduction), n'échappe pas à la règle et subit également cette variation situationnelle (idem), qui introduit une variation au niveau de la langue liée à un registre. Ainsi, l'utilisation de la langue d'un texte relevant de l'ASP peut être établie en fonction de la situation de communication et par le registre auquel il appartient.

Cependant, comme les deux auteurs l'indiquent, l'ASP est également formé par un ensemble de règles d'usage, appelées «normes », qui la configurent (id.). Étant donné la très grande polysémie que recouvre le terme "norme ", il nous paraît primordial de préciser ici que ce dernier sera envisagé dans cette note selon la définition de $\mathrm{C}$. Gledhill et N. Kübler (2016). Sous cet angle, les normes, qui, au fil du temps, peuvent devenir conventionnelles, définissent aussi l'utilisation de la langue.

Ce lien, entre les conventions (les normes) et l'utilisation de la langue déterminée par les registres, présent au sein de la construction de la langue, nous amène à envisager la question suivante : dans quelle mesure les normes établies par les théories et études sur l'ASP influencent-elles les explications fournies sur les registres?

5 La présente note a pour objectif, après avoir défini avec précision la notion non consensuelle de "registre», de présenter une étude de la potentielle influence des normes dans la théorie des registres. Étant donné les nombreuses analyses portant sur le concept complexe de "registre", cette étude se concentre sur deux ouvrages en particulier, sélectionnés en raison de l'influence des analyses de leurs auteurs sur la théorie des registres: Halliday's Introduction to Functional Grammar (Fourth Edition) de Michael A.K. Halliday et Christian M.I.M. Matthiessen (2004), et Register, Genre, and Style de Douglas Biber et Susan Conrad (2009).

\section{Vers une définition du " registre »}

6 Un aperçu de la littérature suffit pour attester que la notion de « registre » ne fait pas consensus au sein de la communauté linguistique. Si ce concept est généralement admis, un véritable flou terminologique autour de ce dernier demeure, tant en anglais qu'en français : en fonction de l'approche théorique choisie, divers autres termes, tels que "genre ", «style » et «type textuel/text type » sont employés au même titre que « registre/register » pour exprimer le même concept (BalaŢchi 2012).

7 Dans le cadre de cette étude, la définition du «registre " qui a été adoptée est celle proposée par la SFL, qui est l'une des approches théoriques s'étant particulièrement intéressées à la question de la variation diaphasique (ou situationnelle [Gadet 1996]). Celle-ci considère la variation langagière comme fonctionnelle, c'est-à-dire comme "résultant de différents contextes situationnels de l'utilisation de la langue» (Neumann $2014: 3$, notre traduction). En effet, comme l'explique également Jeremy Munday :

Halliday's model of discourse analysis, based on what he terms systemic functional linguistics (SFL), [...] sees meaning in the writer's linguistic choices and, through a detailed grammar, systematically relates these choices to the text's function in a wider sociocultural framework [...]. [...] In Halliday's model, importantly, there is a strong interrelation between the linguistic choices, the aims of the communication and the sociocultural framework [...]. (Munday $2016: 142-143$ )

Il résume cette approche un peu plus loin de la façon suivante :

[SFL] links micro-level linguistic choices to the communicative function of a text and the sociocultural meaning behind it. (Munday op.cit. : 165) 
Cette variation est également intéressante à étudier du point de vue des normes étant donné que, d'une part, comme indiqué dans l'appel à contributions à ce numéro de la revue ASp, les normes standardisent les modes de fonctionnement, et, d'autre part, la répétition de situations particulières détermine cette utilisation (Neumann op. cit. : 2). Ainsi :

The rationale underlying the concept of register variation is the assumption that people are never totally free in the way they use language, but rather that recurring situations predetermine their use of language. (Neumann op. cit. : 2)

Pour comprendre un texte et les caractéristiques linguistiques qui le composent, il faut donc appréhender son contexte. Celui-ci est défini par trois paramètres sémiotiques : le Champ (la représentation extérieure de l'expérience envisagée dans le texte), la Teneur (les relations entre locuteurs) et le Mode (la forme de communication) (Munday op. cit. : 145). Il s'agit toutefois ici de paramètres abstraits, auxquels il convient d'associer des éléments concrets afin d'en permettre l'étude linguistique (Neumann op. cit.: 16). M.A.K. Halliday distingue trois métafonctions sémantiques associées à ces paramètres (respectivement, idéationnelle, interpersonnelle et textuelle) qui, par leur concrétisation en des traits lexicaux, grammaticaux et syntaxiques, rendent cette étude possible (idem). Les concepts abstraits d'un registre, essentiels à sa construction, peuvent donc être analysés au travers de caractéristiques linguistiques concrètes et observables dans un texte; il devient ainsi réalisable d'appréhender un contexte au travers d'un texte.

11 Étant donné que leur ouvrage fait l'objet d'une analyse ci-dessous, il nous apparaît utile d'ajouter ici que, si elle s'en inspire grandement, la définition du registre envisagée par D. Biber et S. Conrad s'écarte quelque peu de celle proposée par la SFL. En effet, selon les deux auteurs, les traits linguistiques qui caractérisent un registre sont à interpréter comme des éléments permettant la réalisation des fonctions de communication uniquement; ils ne concernent que la lexico-grammaire et ne participent pas à l'organisation d'un texte (2009: 16). Dans leur théorie, la notion de norme doit donc être étudiée au niveau du genre, qui est constitué de traits auxquels il est conventionnellement associé.

$12 \mathrm{Au}$ vu des distinctions qui peuvent être relevées entre les définitions qui viennent d'être énoncées, il apparaît clair que chaque analyste doit prendre position face à ces choix définitoires avant de commencer toute étude. Dans le cadre de cette analyse, sur la base des définitions présentées ci-avant, nous envisageons le registre comme une variété de langue associée à un contexte de communication particulier.

13 Comme nous l'avons vu plus haut, l'intérêt que présente l'analyse des registres ne se limite pas à la langue anglaise générale : cette analyse s'avère tout aussi pertinente pour les textes relevant de l'ASP. Rappelons qu'à cet égard, C. Gledhill et N. Kübler soutiennent que les registres font partie des éléments à prendre en considération lors de l'analyse d'un texte (op. cit.: 77). Ils ajoutent également que l'étude de la phraséologie de l'ASP, de plus en plus fréquente grâce à la constitution de corpus en linguistique, tient compte notamment de l'analyse de structures récurrentes des registres (Register Pattern Analysis). Autrement dit, cette dernière permet d'identifier des phénomènes phraséologiques, ou d'étudier :

[...] various types of high-level regularities, which for a particular register [...] correspond to the overall configuration of all the relevant lexico-grammatical and semantic patterns which are conventionally (or potentially) associated with that 
register among a particular community of language users. (Gledhill \& Kübler op. cit.

: 77, nous soulignons) par « registre », la question posée initialement peut dès lors être envisagée : dans la mesure où l'étude des registres consiste en l'étude de l'utilisation de la langue et, par conséquent, où les usages faits de la langue détiennent une place capitale dans la caractérisation et l'emploi des registres, à quel point les normes influencent-elles la théorie des registres? Afin de trouver des réponses à cette question, nous analysons ici deux ouvrages de référence. Bien que ces derniers traitent des registres uniquement (pour celui de M.A.K. Halliday et C.M.I.M. Matthiessen) ou majoritairement (pour celui de D. Biber et S. Conrad) en langue générale, leur examen semble pertinent dans le cadre de cette analyse axée sur l'ASP en raison du continuum, des liens étroits qui unissent ces deux types de langue (Resche 1999, cité dans Gledhill \& Kübler op. cit. : 72).

\subsection{Halliday's Introduction to Functional Grammar (Fourth Edition) de M.A.K. Halliday et C.M.I.M. Matthiessen (2004)}

Étant donné que ce livre vise à présenter exhaustivement les aspects grammaticaux de la langue, notre analyse s'est concentrée spécifiquement sur la définition donnée à la notion de registre. Rappelons que la Grammaire Systémique Fonctionnelle a pour objectif fondamental de «fournir une terminologie grammaticale précise pour ce que l'on appelle 'l'analyse discursive'» (Munday op. cit. : 323, notre traduction). Le concept de registre est défini au début de l'ouvrage : il se trouve dans le sous-chapitre intitulé « Basic concepts for the study of language " appartenant à la partie "The architecture of language ». Le registre s'inscrit dans la catégorie des « exemplifications » (instantiations). Pour comprendre l'organisation de la langue, selon M.A.K. Halliday et C.M.I.M. Matthiessen, il faut prendre en considération deux perspectives : la langue fonctionne en tant que système et en tant que texte (2004: 27). L'exemplification permet alors de lier ces deux perspectives :

The concept we need here is that of instantiation. The system of a language is

'instantiated' in the form of text. (Halliday \& Matthiessen op. cit. : 27)

Autrement dit, pour reprendre le raisonnement des deux linguistes, un texte n'est qu'un exemple du système, du potentiel sémantique de la langue concernée, et, à l'inverse, le système est représenté dans la réalité par le texte. Ce système peut être divisé en sous-catégories déterminées par des textes partageant des caractéristiques communes. Ces dernières (nommées patterns) permettent ainsi d'élaborer différents types textuels, qu'on pourrait comparer a priori sur la base de toute strate de la langue (sémantique, lexico-grammaire, phonologie et phonétique). Toutefois, les textes 
varient en fonction du contexte dans lequel ils sont utilisés. Ces sous-catégories sont dès lors définies par les registres, c'est-à-dire :

[...] patterns of instantiation of the overall system associated with a given type of context (a situation type). (Halliday \& Matthiessen op. cit. : 29)

En d'autres termes, c'est le contexte, le cadre d'utilisation de la langue, qui détermine les caractéristiques des différentes strates de la langue. Sous cet angle, il nous paraît approprié de lier cette notion de contexte à celle des usages de la langue. La langue, dont le système est divisé par les registres, est déterminée par les usages.

Qu'en est-il des normes, des règles d'usage, dans cette théorie ? Pour observer leur influence, il faut revenir à la thèse précédente des auteurs selon laquelle la langue est composée de strates: deux strates véhiculant le contenu (la sémantique et la lexicogrammaire) et deux strates en permettant son expression (la phonologie et la phonétique). Elles sont couplées dans la mesure où les strates dont la fonction est d'organiser la langue (la lexico-grammaire et la phonologie) sont associées aux strates qui ont pour fonction de permettre au locuteur d'interagir avec son environnement (la sémantique et la phonétique). Ces relations de réalisation de la langue entre chaque couple sont, d'après M.A.K. Halliday et C.M.I.M. Matthiessen, naturelles plutôt que conventionnelles. En revanche, la relation entre le contenu et l'expression (plus spécifiquement entre la lexico-grammaire et la phonologie) est quant à elle principalement conventionnelle. Elle a été en effet choisie arbitrairement. Il est donc intéressant de noter ici que, finalement, d'après cette partie de l'ouvrage, l'accent est mis sur le contexte d'utilisation; les normes ne sont visiblement pas un critère dans la définition des registres.

\subsection{Register, Genre, and Style de D. Biber et S. Conrad (2009)}

Comme indiqué dans l'introduction de nos propos, ce manuel ne traite pas uniquement de l'anglais général: il se penche en effet notamment sur la question de l'écriture universitaire (academic prose), qui a été considérée ici comme un ASP au vu de la définition donnée ci-dessus. D. Biber et $\mathrm{S}$. Conrad analysent deux types d'écriture universitaire en particulier (l'article de recherche et les manuels scolaires), dont la configuration est influencée, selon eux, par les besoins du lecteur et par les fonctions et les objectifs de communication. Notre étude est concentrée sur la description de l'article de recherche. L'ouvrage énonce une série de caractéristiques linguistiques présentes dans l'article de recherche rédigé en anglais (le passif, la nominalisation, etc.). Dans le cadre de l'analyse de cet ouvrage, la notion de norme se situe non pas dans la définition du registre, mais dans celle du genre. En effet, D. Biber et S. Conrad adoptent une définition quelque peu différente du registre, qu'ils considèrent comme une "variété de langue associée à la fois à une situation particulière d'utilisation et à des caractéristiques linguistiques largement répandues qui permettent la réalisation de fonctions importantes dans ce contexte d'utilisation» (2009: 31, notre traduction). L'analyse de la structure d'un texte et des éléments conventionnellement associés au type de ce texte réside dans l'analyse de son genre, et non de son registre (op. cit. : 16). La notion de norme sera alors à chercher au niveau du genre. Dans le cas de l'article de recherche, les auteurs font une description spécifique des usages du modèle IMRaD (acronyme utilisé pour "Introduction, Methods, Results, and Discussion») réalisés en fonction des normes : 
[From a genre perspective,] traditional (experimental) research articles are one of the clearest examples of a variety that is structured according to textual conventions [since] [n]early all experimental research articles have the same overall organization [...] [whose] components are expected to occur in the text, and always in the same order. (Biber \& Conrad op. cit. : 131)

C'est donc, d'après cette théorie, avant tout la structure qui suit des conventions, des normes. Certains traits linguistiques (comme le sous-titre "Introduction») sont également choisis, car ils ont été conventionnellement fixés et associés à un genre textuel particulier.

Leur analyse de l'article de recherche se poursuit par une étude diachronique (du XVII siècle à nos jours) de ce type de registre, qui a fortement changé au fil du temps. Après une analyse sur corpus, les deux auteurs concluent que les conventions textuelles et les traits linguistiques des articles de recherche ont évolué pour s'adapter aux objectifs communicationnels et aux lecteurs. Autrement dit, les lecteurs, et de manière plus large la société, ont changé, entraînant ainsi des changements au niveau des objectifs de communication qui ont, à leur tour, eu des répercussions sur les conventions textuelles et sur les traits linguistiques. Ce registre a ainsi vu ses normes être influencées par les usages, eux-mêmes formés par le contexte. L'usage semble dans ce cas-ci influer fortement sur la norme.

\section{Réflexions sur une approche des normes en traduction}

23 Notre formation de traducteur et les liens étroits qu'entretiennent la linguistique contrastive et la traductologie (Vandepitte \& De Sutter 2013) nous amènent à terminer cette analyse en abordant cette question sous un angle traductif. C. Gledhill et N. Kübler soulignent d'ailleurs l'importance de l'analyse des registres au cours de la traduction de textes spécialisés (2016: 80), comme l'affirmaient déjà Hélène Chuquet et Michel Paillard (1987) dans le cadre de traduction de textes " généraux »:

Avant de traduire un texte, il importe d'identifier le registre auquel il appartient [...].

(Chuquet \& Paillard op. cit. : 216)

24 À cette fin, la célèbre théorie des normes en traduction de Gideon Toury dans les années 1990 (Munday op. cit. : 174) est sans nul doute capitale. Notre intention étant avant tout de cerner les notions de norme et d'usage dans des théories linguistiques et traductologiques, nous ne traitons pas ici de toute la théorie de G. Toury, ni des débats qu'elle a suscités; nous envisageons brièvement l'introduction du livre Translation and Norms de Christina Schäffner (1999), qui résume cette théorie et la notion de norme en traduction d'une manière qui nous a amenée à les envisager dans cette note, bien que les registres ne constituent pas l'objet principal de cette description.

D'après C. Schäffner, la norme comporte les qualités de ce qui est correct (correctness) et approprié (appropriateness). Lorsqu'il traduit un texte, le traducteur doit tenir compte de ce qu'il peut faire avec la langue et de ce qu'il convient de faire dans un contexte donné, décrit par les conventions, les normes, comprises comme des "standards ou modèles de comportement correct ou approprié et de produits corrects ou appropriés issus de ce comportement » (op. cit. : 5, notre traduction). Comme l'avance C. Schäffner, la traductologie avait pour habitude de fournir des règles normatives en vue de recontextualiser le texte original dans la langue cible et ainsi de prendre en 
considération les normes des langues source et cible ainsi que les normes textuelles. La position de G. Toury est, quant à elle, présentée de la manière suivante :

Translational norms are understood as internalised behavioural constraints which embody the values shared by a community. (Schäffner op. cit. : 5)

Par conséquent, outre les systèmes langagiers, ces normes influencent les décisions prises lors du processus de traduction. C. Schäffner conclut son introduction par deux dichotomies intéressantes :

As far as linguistic norms are concerned, they are usually codified in an institutionalised way, for example in grammar books and lexicons. In translation studies, scholars have attempted to set up normative translation laws and translation rules or guidelines [...] meant both to explain translation regularities and to predict certain structures (thus limiting choices) [...]. In these contexts, however, 'normative' is meant in the sense of 'prescriptive'. In its sense of 'descriptive' it refers to the study of the norms themselves or to the study of [, according to A. Chesterman,] the 'products, processes or behaviour that are taken to constitute or represent the norms' (Chesterman, 1993: 11). (Schäffner op. cit. : 7)

Ainsi, si la linguistique tend à codifier ses normes au niveau institutionnel, la traductologie met en place des normes prescriptives. Comme C. Schäffner le souligne, l'idée que la traduction puisse être considérée comme un «comportement gouverné par des normes " peut soulever de nombreuses questions, dont certaines ont déjà fait l'objet de diverses analyses. Plusieurs d'entre elles ont retenu notre attention, et nous avons souhaité les retranscrire ici étant donné les liens qui peuvent être établis entre les normes en ASP et celles de la traduction spécialisée :

How do translators acquire norms, do they behave according to norms, and are they conscious of their norm-governed behaviour? [...] Are there translation specific norms, or more general norms in society that also influence translational behaviour? [...] Is the behaviour of translators indeed governed by norms, or are they rather actively involved in the maintenance of norms [...] [ ?] (Schäffner op. cit. :7)

\section{Conclusion}

Étant donné que la théorie des registres a pour objectif de présenter le fonctionnement de l'utilisation de la langue, la présence de règles d'usage, de normes, tant en anglais général qu'en ASP, dans ce type de théorie pouvait a priori paraitre peu discutable. Après une analyse préliminaire, avant tout exploratoire, de la théorie sur les registres de deux grands ouvrages, il s'avère néanmoins difficile d'affirmer de tels propos. En effet, ces deux livres font peu référence à la notion de « norme » (appelée alors souvent " convention ») lorsqu'ils traitent des registres. L'analyse révèle également que, dans la définition des registres de la théorie de M.A.K. Halliday et C.M.I.M Matthiessen, fondatrice de la SFL, la norme se retrouve uniquement dans la relation de réalisation entre le contenu d'un message et son expression. Notons cependant que, cet ouvrage se concentrant principalement sur l'analyse de structures lexico-grammaticales, il serait intéressant d'envisager l'étude d'autres approches de la SFL qui mettent les contextes culturels et situationnels davantage en avant. L'on pourrait également se pencher sur l'approche de Jim R. Martin et David Rose (2007), dont la contribution en SFL est majeure, ou sur la théorie de Critical Discourse Analysis, qui met en avant la dimension sociale de la communication et l'usage de la langue (Petitclerc 2009). La définition fournie par D. Biber et S. Conrad, quant à elle, situe cette notion au niveau du genre et 
non du registre. La norme dépasse l'usage, sauf lorsque la dimension diachronique est ajoutée à l'analyse. Cette dimension temporelle semble soulever de nouveaux questionnements en matière de normes et d'usages en ASP. Par exemple, en quoi consistent concrètement les changements sociétaux considérés plus haut comme sources de modifications des objectifs de communication et donc des registres? De quel(s) autre(s) aspect(s) de la langue ces changements ont-ils été à l'origine ? En quoi diffèrent les changements lexico-grammaticaux et syntaxiques relevés au cours de l'analyse de D. Biber et $\mathrm{S}$. Conrad entre textes appartenant à des ASP différents (médical, juridique, etc.) ? Enfin, s'ajoutent à ces questions d'autres interrogations que la présente analyse sur les normes et les registres peut susciter tant en linguistique qu'en traduction : le degré d'influence exercée par les normes et les usages change-t-il en fonction des catégories linguistiques étudiées (collocations, phraséologie, terminologie, etc.) ? Qu'en est-il au niveau de la langue ? Observe-t-on des différences d'influence entre l'ASP et le français de spécialité ? La présence de la notion de norme dans des écrits théoriques est-elle d'ordre culturel? Les questions soulevées par C. Schäffner concernant ces "normes traductives » mériteraient d'être aussi davantage envisagées, car elles permettraient peut-être de mettre également au jour des mécanismes internes de la traduction et de la linguistique, de déceler plus de liens entre ces deux disciplines à cet égard (comment le traducteur gère-t-il son travail entre normes linguistiques et normes traductives ?), de se pencher sur l'enseignement de la traduction (l'apprenant en traduction a-t-il conscience des normes? Quel type de normes lui enseigner ? Comment et quand faut-il le faire ?), etc. Les réflexions à ce sujet sont manifestement nombreuses et peuvent vraisemblablement ouvrir la voie à de nouvelles pistes de recherche.

\section{BIBLIOGRAPHIE}

BALAŢCHI, Raluca-Nicoleta. 2012. «Style et registre de langue en traduction ». Écho des Études Romanes 8/2, 15-25, consulté le 7/10/2016 <http://www.eer.cz/files/2012-2/2012-2-2Balatchi.pdf>.

BIBER, Douglas \& Susan CONRAD. 2009. Register, Genre, and Style. Cambridge/New York : Cambridge University Press.

CHUQUET, Hélène \& Michel PAILLARD. 1987. Approche linguistique des problèmes de traduction. Paris : Ophrys.

GADET, Françoise. 1996. « Niveaux de langue et variations intrinsèques ». In BENSIMON, P. (dir.), Palimpsestes 10 : Niveaux de langue et registres de la traduction. Paris: Presses de la Sorbonne Nouvelle, 17-39.

GLEDHILL, Christopher \& Natalie KÜBLER. 2016. « What can linguistic approaches bring to English for Specific Purposes? ». ASp 69, 65-95.

HALLIDAY, Michael A.K. \& Christian M.I.M. MATTHIESSEN. 2004. Halliday's Introduction to Functional Grammar (Fourth Edition). Oxon/New York : Routledge. 
MARTIN, Jim R. \& David ROSE. 2007. Working with Discourse. Meaning beyond the Clause (Second Edition). Londres/New York : Bloomsbury Academic.

MUNDAY, Jeremy. 2016. Introducing Translation Studies: Theories and Applications (Fourth Edition). Oxon/New York : Routledge.

NEUMANN, Stella. 2014. Contrastive Register Variation. Berlin/Boston : De Gruyter.

PETITCLERC, Adèle. 2009. «Introduction aux notions de contexte et d'acteurs sociaux en Critical Discourse Analysis ». Semen 27, 17-32, consulté le 04/07/2017 <https://semen.revues.org/8540>. SCHÄFFNER, Christina. 1999. Translation and Norms. Clevedon : Multilingual Matters LTD (coll. Current Issues in Language and Society).

VANDEPITTE, Sonia \& Gert DE SUTTER. 2013. « Contrastive linguistics and translation studies ». In GAMBIER, Y. \& L. VAN DOORSLAER (dir.), Handbook of Translation Studies (volume 4). Amsterdam/ Philadelphie : John Benjamins Publishing Company, 36-41.

\section{RÉSUMÉS}

Étant donné que l'anglais de spécialité (ASP) se construit sur la base de normes langagières, liées à des conventions, et qu'il se décline en fonction de registres tels que définis par la linguistique systémique fonctionnelle (SFL), qui déterminent sa lexico-grammaire et ses usages, la question se pose de savoir dans quelle mesure les normes interviennent dans les explications théoriques des registres en ASP. Cette note présente l'étude de deux ouvrages de référence sur l'analyse du discours afin d'apporter des éléments de réponse à cette question. Elle fait apparaître que, dans ces deux livres, les notions de norme et d'usage sont rarement mentionnées et, quand elles le sont, la norme semble l'emporter sur l'usage, sauf lorsque la dimension diachronique est prise en compte, ouvrant dès lors la voie à de nouvelles perspectives d'analyse. La note se conclut par une approche des normes en traduction en vue de fournir de nouvelles pistes de recherche.

Since English for Specific Purposes (ESP) is made up of rules of language called 'norms' and since it is shaped by a register, which, according to the Systemic Functional Linguistics theory (SFL), determines its lexico-grammar and language use, an analysis of the potential influence of norms on register theory may be of interest to the study of ESP. This note examines two reference books on discourse analysis in order to explore the relationship between norms and language use. This study shows that those books rarely mention the notions of norm and practice in the description of register and, when they do, norms seem to outweigh practice, except when the diachronic dimension is taken into account. It may therefore be interesting to initiate further investigation on this dimension. The note ends with an approach to the concept of translational norms and suggests new research paths.

\section{INDEX}

Mots-clés : linguistique systémique fonctionnelle, norme, registre, SFL, traduction

Keywords : norm, register, SFL, systemic functional linguistics, translation 


\section{AUTEUR}

\section{TIFFANY JANDRAIN}

Tiffany Jandrain est doctorante au sein du Service de Traduction spécialisée et Terminologie à la Faculté de Traduction et d'Interprétation - École d'Interprètes Internationaux (FTI - EII) de l'Université de Mons en Belgique. Après avoir obtenu à la FTI - EII les diplômes de Master en traduction et de Master de spécialisation en linguistique appliquée, elle mène des recherches portant sur la transposition des registres en traduction spécialisée de l'anglais vers le français à des fins pédagogiques sous la direction de Christine Michaux et de Gudrun Vanderbauwhede. Ses principaux domaines de recherche concernent la traduction spécialisée, la linguistique contrastive et la linguistique appliquée ; ses langues de travail sont l'anglais, le russe et le néerlandais. tiffany.jandrain@umons.ac.be 